\title{
ELEMENTARY PROOFS \\ OF THE ABSTRACT PRIME NUMBER THEOREM FOR ALGEBRAIC FUNCTION FIELDS
}

\author{
WEN-BIN ZHANG
}

\begin{abstract}
Elementary proofs of the abstract prime number theorem of the form $\Lambda(m)=q^{m}+O\left(q^{m} m^{-1}\right)$ for algebraic function fields are given. The proofs use a refinement of a tauberian theorem of Bombieri.
\end{abstract}

\section{INTRODUCTION}

The main purpose of this paper is to give elementary proofs of the abstract prime number theorem for algebraic function fields (henceforth, the P.N.T.) which was established in the author's paper [8]. In the analytic argument in [8], the fact that the generating function $Z^{\#}(y)$ has no zeros on the circle $|y|=q^{-1}$ plays an important role. In this paper, we shall show that the nonvanishing of the generating function has an elementary interpretation which can be expressed as another analogue (see (2.3)) of Selberg's formula in classical prime number theory. In a recent paper [2], Bombieri also proposed this formula. By introducing the new analogue and proving an elegant tauberian theorem (see Theorem 3.1), he made a correction to the last part of the argument in his earlier paper [1]. In this paper, we shall refine this tauberian theorem in a quantitative form. The result, Theorem 3.2, is sharp in some sense. Combining our elementary proof of the new analogue of Selberg's formula and the new tauberian theorem will give an elementary proof of the P.N.T. with a remainder term (see Theorems 4.1 and 4.2). Since the last part of the argument given in [1] is inadequate to prove the P.N.T., it is an interesting question whether the P.N.T. (or the conclusion in [1]) can be established with the aid of the new analogue mentioned above. We shall show briefly that this is indeed the case.

The author thanks Professor E. Bombieri for the preprint of his article [2].

\section{AN ELEMENTARY PROOF OF THE FIRST ANALOGUE OF SELBERG'S FORMULA}

We begin by recalling the concept of an additive arithmetic semigroup, which was developed by Knopfmacher [6]. An additive arithmetic semigroup is, by

Received by the editors November 21, 1989 and, in revised form, June 11, 1990.

1980 Mathematics Subject Classification (1985 Revision). Primary 11N80; Secondary 11T41, $11 \mathrm{~T} 55$.

Key words and phrases. Abstract prime number theorem, additive arithmetic semi-group, additive convolution, tauberian theorem. 
definition, a free commutative semigroup $\mathscr{G}$ with identity element 1 such that $\mathscr{G}$ has a countable free generating set $\mathscr{P}$ of "primes" $p$ and such that $\mathscr{G}$ admits an integer-valued degree mapping $\partial: \mathscr{G} \rightarrow N \cup\{0\}$ satisfying:

(i) $\partial(1)=0$ and $\partial(p)>0$ for all $p \in \mathscr{P}$,

(ii) $\partial(a b)=\partial(a)+\partial(b)$ for all $a, b \in \mathscr{G}$, and

(iii) the total number $G(n)$ of elements of degree $n$ in $\mathscr{G}$ is finite for each $n \geq 0$.

Let $P(n)$ denote the total number of primes of degree $n$ in $\mathscr{G}$. We set

$$
\Lambda(m)=\sum_{\substack{p \in \mathscr{P}, r \geq 1 \\ \partial\left(p^{\prime}\right)=m}} \partial(p), \quad m=0,1,2, \ldots,
$$

which is the counterpart of the number $N_{m}$ for an algebraic curve $C$ in [1]. Then,

$$
\Lambda(n)=\sum_{r \mid n} \frac{n}{r} P\left(\frac{n}{r}\right) .
$$

The arithmetic function $G(n)$ is an elementary combinatorial function of $P(n)$. This can be expressed explicitly by

$$
\sum_{n=0}^{\infty} G(n) y^{n}=\prod_{m=1}^{\infty}\left(1-y^{m}\right)^{-P(m)} .
$$

Therefore, for brevity, we often use formal power series and formal infinite products to establish identities and simple estimates which can also be obtained directly. Thus, from (1.1), we can deduce

$$
\sum_{n=1}^{\infty} \Lambda(n) y^{n} \sum_{n=0}^{\infty} G(n) y^{n}=\sum_{n=0}^{\infty} n G(n) y^{n} .
$$

Moreover, we shall appeal only to the convergence of real power series but not use analytic behavior. This is the sense in which our argument is elementary.

For brevity, we shall also use additive convolution of arithmetic functions. Let $f(n)$ and $g(n)$ be two arithmetic functions defined for all nonnegative integers. The function $h(n)$ defined by setting

$$
h(n)=\sum_{k=0}^{n} f(k) g(n-k), \quad n=0,1,2,3, \ldots,
$$

is called the additive convolution of $f$ and $g$ and denoted by $f * g$. It is easy to see that additive convolution is associative and commutative. We also define a differentiation operator $L$ on arithmetic functions $f$ by setting $(L f)(n)=$ $n f(n), n=0,1,2, \ldots$. Thus, $(1.2)$ can be rewritten as

$$
\Lambda * G=L G .
$$

The following estimate quoted from [8] was established by using (1.3).

Lemma 1.1. Suppose there exist constants $A>0, q>1$, and $\gamma>1$ such that

$$
G(n)=A q^{n}+O\left(q^{n} n^{-\gamma}\right), \quad n=1,2, \ldots
$$


Then

$$
\sum_{k=1}^{n} \frac{\Lambda(k)}{q^{k}}=n+O(1)
$$

We next give a simpler elementary proof of the following result [1].

Theorem 1.2 (abstract Selberg-Bombieri formula). Suppose there exist constants $A>0, q>1, \gamma>3$, and $c>0$ such that

$$
\left|G(n)-A q^{n}\right| \leq c q^{n} / n^{\gamma}, \quad n=1,2, \ldots .
$$

Then, as $m \rightarrow \infty$,

$$
m \Lambda(m)+\sum_{r=1}^{m-1} \Lambda(r) \Lambda(m-r)=2 m q^{m}+O\left(q^{m}\right)
$$

where the " $O$ " constant depends only on $A, q, \gamma$, and $c$ in (1.4).

Remark. This analogue of Selberg's formula in classical prime number theory was first proved by Bombieri [1] for an algebraic curve $C$. Knopfmacher [6] established this formula for an additive arithmetic semigroup with stronger hypotheses by an elementary argument. Here we shall give a simpler elementary proof. In the following proof, we follow the general idea of Diamond [4] for proving Selberg's formula and make use of the additive convolution techniques.

Proof. The starting-point of our proof is the following convolution identity $(L \Lambda+\Lambda * \Lambda) * G=L^{2} G$, which can be deduced easily from (1.3). Therefore,

$$
L \Lambda+\Lambda * \Lambda=L^{2} G * G^{-1},
$$

where $G^{-1}$ is the additive convolution inverse of $G$. Since

$$
\sum_{n=0}^{\infty} G^{-1}(n) y^{n}=\prod_{m=1}^{\infty}\left(1-y^{m}\right)^{P(m)},
$$

it is plain that $\left|G^{-1}(n)\right| \leq G(n)$.

The arithmetic function $L^{2} G$ can be approximated well by a function of the form

$$
2(G * T * T)+c_{2}(G * T)+c_{3} G,
$$

where the function $T$ is defined by setting $T(n)=q^{n}, n=0,1,2, \ldots$, and the coefficients $c_{2}$ and $c_{3}$ will be specified later. We denote

$$
G(n)=A q^{n}+a_{n} q^{n} n^{-\gamma}, \quad n=1,2, \ldots,
$$

where $\left|a_{n}\right| \leq c$ by (1.4). Then we have

$$
G * T(n)=q^{n}\left\{A n+\left(1+\sigma_{1}\right)+O\left(\frac{1}{(n+1)^{\gamma-1}}\right)\right\}
$$

where $\sigma_{1}=\sum_{s=1}^{\infty} a_{s} s^{-\gamma}$, and

$$
\begin{aligned}
G * T * T(n) & =\sum_{0 \leq s \leq n} G(s)(T * T)(n-s) \\
& =(n+1) q^{n}+\sum_{1 \leq s \leq n}(n-s+1) q^{n-s}\left(A q^{s}+a_{s} q^{s} s^{-\gamma}\right) \\
& =q^{n}\left\{\frac{1}{2} A n(n+1)+(n+1)\left(1+\sigma_{1}\right)-\sigma_{2}+O\left(\frac{1}{(n+1)^{\gamma-2}}\right)\right\},
\end{aligned}
$$


where $\sigma_{2}=\sum_{s=1}^{\infty} a_{s} s^{-\gamma+1}$. If we introduce $R=L^{2} G-2 G * T * T-c_{2} G * T-c_{3} G$ and choose

$$
c_{2}=-1-\frac{2}{A}\left(1+\sigma_{1}\right), \quad c_{3}=\frac{1}{A}\left\{-\left(2+c_{2}\right)\left(1+\sigma_{1}\right)+2 \sigma_{2}\right\},
$$

then we have

$$
\begin{aligned}
R(n)= & q^{n}\left\{-\left(2\left(1+\sigma_{1}\right)+A+c_{2} A\right) n\right. \\
& \left.\quad+\left(-\left(2+c_{2}\right)\left(1+\sigma_{1}\right)+2 \sigma_{2}-c_{3} A\right)+O\left(n^{-\gamma+2}\right)\right\} \\
= & q^{n} O\left(n^{-\gamma+2}\right) .
\end{aligned}
$$

We now rewrite (1.6) as $L \Lambda+\Lambda * \Lambda=2 T * T+c_{2} T+c_{3} \delta+R * G^{-1}$, where $\delta$ is the additive convolution identity defined by

$$
\delta(n)= \begin{cases}1, & \text { if } n=0, \\ 0, & \text { if } n>0\end{cases}
$$

Then we have

$$
m \Lambda(m)+\sum_{r=1}^{m-1} \Lambda(r) \Lambda(m-r)=2 m q^{m}+O\left(q^{m}\right)
$$

since, by (1.7),

$$
\left|R * G^{-1}(m)\right|=\left|\sum_{s=0}^{m} R(s) G^{-1}(m-s)\right| \ll q^{m}\left(1+\sum_{s=1}^{m} s^{-\gamma+2}\right) \ll q^{m} .
$$

\section{AN ELEMENTARY PROOF OF THE SECOND ANALOGUE OF SELBERG'S FORMULA}

In this section, we shall give an elementary proof of (2.3) below which we call the second analogue of Selberg's formula. We shall also show that (2.3) is in fact an "elementary version" of nonvanishing of the generating function $Z^{\#}(y)$ (see [8]).

Theorem 2.1. Suppose there exist constants $A>0, q>1$ such that

$$
\sum_{n=1}^{\infty}\left(G(n)-A q^{n}\right)^{2} q^{-n}<\infty
$$

Then

$$
\sum_{s=1}^{n} \frac{\Lambda(2 s)}{q^{2 s}}=n+O(1)
$$

and

$$
n \Lambda(2 n)+\sum_{s=1}^{n} \Lambda(2 s) \Lambda(2 n-2 s)=2 n q^{2 n}+O\left(q^{2 n}\right) .
$$

Remark 1. Bombieri also arrived at (2.3) for an algebraic curve $C$ in his recent paper [2].

Remark 2. (2.2) is a consequence of (2.3). However, (2.2) can be established with a weaker condition than that needed for $(2.3)$, as we can see from Lemma 2.2.

The proof of Theorem 2.1 follows the general idea for proving Theorem 3.1 in [8]. We first prove several lemmas. 
Lemma 2.2. Let

$$
H(n)=\sum_{k=0}^{n}(-1)^{k} G(k) G(n-k) .
$$

If there exist constants $A_{1}>0$ and $\gamma>1$ such that

$$
H(2 n)=A_{1} q^{2 n}+O\left(q^{2 n} n^{-\gamma}\right) \text { as } n \rightarrow \infty
$$

then (2.2) holds. Furthermore, if (2.5) with $\gamma>3$ is true then (2.3) holds.

Remark. Plainly, $H(2 n-1)=0, n=1,2, \ldots$.

Proof. We first note that

$$
\begin{aligned}
\sum_{n=0}^{\infty} H(n) y^{n} & =\sum_{n=0}^{\infty}(-1)^{n} G(n) y^{n} \sum_{n=0}^{\infty} G(n) y^{n} \\
& =\prod_{\substack{m=1 \\
m \text { even }}}^{\infty}\left(1-y^{m}\right)^{-2 P(m)} \prod_{\substack{m=1 \\
m \text { odd }}}^{\infty}\left(1-y^{2 m}\right)^{-P(m)} \\
& =\prod_{m=1}^{\infty}\left(1-y^{m}\right)^{-\bar{P}(m)}
\end{aligned}
$$

where

$$
\bar{P}(m)= \begin{cases}0, & \text { if } m \text { is odd } \\ 2 P(m)+P(m / 2), & \text { if } m=2 k \text { with } k \text { odd } \\ 2 P(m), & \text { if } 4 \mid m .\end{cases}
$$

Therefore, $H(n) \geq 0$ for all $n$.

We define arithmetic functions $H_{1}$ and $\Lambda_{1}$ by setting $H_{1}(n)=H(2 n)$ and $\Lambda_{1}(n)=\Lambda(2 n)$. Then we have $H_{1}(n)=A_{1}\left(q^{2}\right)^{n}+O\left(\left(q^{2}\right)^{n} n^{-\gamma}\right)$ with $A_{1}>0$ and $q^{2}>1$ by (2.5). It is easy to verify that

$$
\begin{aligned}
& \sum_{n=1}^{\infty} \Lambda_{1}(n) y^{n} \sum_{n=0}^{\infty} H_{1}(n) y^{n} \\
&=\frac{1}{2}\left\{\sum_{n=1}^{\infty} \Lambda(n)\left(y^{1 / 2}\right)^{n}+\sum_{n=1}^{\infty} \Lambda(n)\left(-y^{1 / 2}\right)^{n}\right\} \sum_{n=0}^{\infty} G(n)\left(y^{1 / 2}\right)^{n} \\
& \cdot \sum_{n=0}^{\infty} G(n)\left(-y^{1 / 2}\right)^{n}=\sum_{n=0}^{\infty} n H_{1}(n) y^{n},
\end{aligned}
$$

or, equivalently

$$
\Lambda_{1} * H_{1}=L H_{1} \text {. }
$$

Therefore, if (2.5) with $\gamma>1$ is true, then, by Lemma 1.1,

$$
\sum_{k=1}^{n} \frac{\Lambda_{1}(k)}{\left(q^{2}\right)^{k}}=\sum_{k=1}^{n} \frac{\Lambda(2 k)}{q^{2 k}}=n+O(1) .
$$

Furthermore, if (2.5) with $\gamma>3$ is true, then, by Theorem 1.2,

$$
n \Lambda_{1}(n)+\sum_{s=1}^{n-1} \Lambda_{1}(s) \Lambda_{1}(n-s)=2 n q^{2 n}+O\left(q^{2 n}\right)
$$

and (2.3) follows. 
Lemma 2.3. Suppose there exist constants $A>0, q>1$, and $\nu$ with $0 \leq \nu<1$ such that

$$
\sum_{n=1}^{\infty}\left(G(n)-A q^{n}\right)^{2} q^{-2 \nu n}<\infty
$$

Then there exists a constant $A_{1}$ such that

$$
H(2 n)=A_{1} q^{2 n}+o\left(q^{2 \nu n}\right) \text { as } n \rightarrow \infty .
$$

Proof. We denote $G(n)=A q^{n}+a_{n} q^{\nu n}, n=1,2, \ldots$ Then, $\sum_{n=1}^{\infty} a_{n}^{2}<\infty$ and $a_{n} \rightarrow 0$ as $n \rightarrow \infty$. We first show that

$$
\sum_{k=1}^{2 m-1}(-1)^{k} a_{k} a_{2 m-k}=o(1) \quad \text { as } m \rightarrow \infty .
$$

Actually, we have

$$
\sum_{k=1}^{2 m-1}(-1)^{k} a_{k} a_{2 m-k}=2 \sum_{k=1}^{m-1}(-1)^{k} a_{k} a_{2 m-k}+(-1)^{m} a_{m}^{2}
$$

and by the Cauchy-Schwarz inequality,

$$
\left|\sum_{k=1}^{m-1}(-1)^{k} a_{k} a_{2 m-k}\right| \leq\left(\sum_{k=1}^{m-1} a_{k}^{2}\right)^{1 / 2}\left(\sum_{k=1}^{m-1} a_{2 m-k}^{2}\right)^{1 / 2} \rightarrow 0 .
$$

Then we have

$$
\begin{aligned}
H(2 m)= & 2 G(2 m)+\sum_{k=1}^{2 m-1}(-1)^{k} G(k) G(2 m-k) \\
= & 2\left(A q^{2 m}+a_{2 m} q^{2 \nu m}\right) \\
& +\sum_{k=1}^{2 m-1}(-1)^{k}\left(A q^{k}+a_{k} q^{\nu k}\right)\left(A q^{2 m-k}+a_{2 m-k} q^{\nu(2 m-k)}\right) \\
= & \left(2 A-A^{2}\right) q^{2 m}+2 A q^{2 m} \sum_{k=1}^{2 m-1}(-1)^{k} a_{k} q^{-(1-\nu) k} \\
& +q^{2 \nu m} \sum_{k=1}^{2 m-1}(-1)^{k} a_{k} a_{2 m-k}+2 a_{2 m} q^{2 \nu m} \\
= & \left(2 A-A^{2}+2 A \sigma\right) q^{2 m}+o\left(q^{2 \nu m}\right),
\end{aligned}
$$

where $\sigma=\sum_{k=1}^{\infty}(-1)^{k} a_{k} q^{-(1-\nu) k}$, by (2.9).

Lemma 2.4. Assume the hypothesis of Theorem 2.1. Then we have $A_{1}>0$ in Lemma 2.3.

Proof. We note that $A_{1} \geq 0$ since all $H(n) \geq 0$. Suppose the conclusion of the lemma is not true so that $A_{1}=0$. Hence, by Lemma 2.3,

$$
H(2 n)=o\left(q^{n}\right)
$$


Therefore, we have $\sum_{n=0}^{\infty} H(n) q^{-n}=B>0$, since $H(n)$ are not all zeros. Define arithmetic functions $G_{1}$ and $G_{2}$ by setting

$$
\sum_{n=0}^{\infty} G_{1}(n) y^{n}=\prod_{\substack{m=1 \\ m \text { odd }}}^{\infty}\left(1-y^{m}\right)^{-P(m)},
$$

and

$$
\sum_{n=0}^{\infty} G_{2}(n) y^{n}=\prod_{\substack{m=1 \\ m \text { even }}}^{\infty}\left(1-y^{m}\right)^{-P(m)}
$$

Then, plainly, $0 \leq G_{1}(n) \leq G(n)$ and $0 \leq G_{2}(n) \leq G(n)$. Therefore, $\sum_{n=0}^{\infty} G_{1}(n) q^{-2 n}$ converges. From (2.6), (2.11), and (2.12), we have

$$
\left(\sum_{n=0}^{\infty} G_{2}(n) y^{n}\right)^{2}\left(\sum_{n=0}^{\infty} G_{1}(n) y^{2 n}\right)=\sum_{n=0}^{\infty} H(n) y^{n}
$$

It follows that $\sum_{n=0}^{\infty} G_{2}(n) q^{-n}$ converges and $\sum_{n=0}^{\infty} G_{2}(n) q^{-n}=B_{1}>0$. We now note that

$$
\sum_{n=0}^{\infty} G_{1}(n) y^{n} \sum_{n=0}^{\infty} G_{2}(n) y^{n}=\sum_{n=0}^{\infty} G(n) y^{n} .
$$

Therefore,

$$
\begin{aligned}
\lim _{y \rightarrow q^{-1}-}(1-q y) \sum_{n=0}^{\infty} G_{1}(n) y^{n} & =\lim _{y \rightarrow q^{-1}-} \frac{(1-q y) \sum_{n=0}^{\infty} G(n) y^{n}}{\sum_{n=0}^{\infty} G_{2}(n) y^{n}} \\
& =\frac{A}{B_{1}}=B_{2}>0 .
\end{aligned}
$$

This implies that

$$
\limsup _{n \rightarrow \infty} G_{1}(n) q^{-n} \geq B_{2}>0 .
$$

If we define $G_{3}$ and $G_{4}$ by setting

$$
\sum_{n=0}^{\infty} G_{3}(n) y^{n}=\sum_{n=0}^{\infty} G_{1}(n) y^{2 n}
$$

and

$$
\sum_{n=0}^{\infty} G_{4}(n) y^{n}=\left(\sum_{n=0}^{\infty} G_{2}(n) y^{n}\right)^{2},
$$

then, from (2.13), we have

$$
H(n)=G_{3}(n)+G_{3}(n-1) G_{4}(1)+\cdots+G_{3}(1) G_{4}(n-1)+G_{4}(n) \geq G_{3}(n)
$$

and hence

$$
\limsup _{n \rightarrow \infty} H(2 n) q^{-n} \geq \underset{n \rightarrow \infty}{\limsup } G_{1}(n) q^{-n}>0
$$

by (2.14). This contradicts (2.10).

Proof of Theorem 2.1. Now, (2.2) and (2.3) follow immediately from Lemmas 2.4 and 2.2 . 
To conclude this section, we show that the formula (2.3) is in fact an "elementary version" of nonvanishing at the point $y=-q^{-1}$ of the generating function

$$
Z^{\#}(y)=\sum_{n=0}^{\infty} G(n) y^{n}=\prod_{m=1}^{\infty}\left(1-y^{m}\right)^{-P(m)}
$$

(see [8]). Actually, $1+\sum_{n=0}^{\infty} H(n) y^{n}=Z^{\#}(y) Z^{\#}(-y)$. From Lemma 3.3 in [8], if $Z^{\#}(y)$ has a zero at $y=-q^{-1}$, then $H(2 n)=o\left(q^{n}\right)$, i.e., $A_{1}=0$ in $(2.7)$. Therefore, from (2.7),

$$
\Lambda(2 n)=\Lambda_{1}(n) \leq \Lambda_{1} * H_{1}(n)=n H_{1}(n)=n H(2 n)=o\left(n q^{n}\right)
$$

and hence (2.3) does not hold. Conversely, if $Z^{\#}(y)$ has no zeros at $y=-q^{-1}$, then plainly $A_{1} \neq 0$ in (2.8) and (2.3) follows.

\section{A TAUBERIAN THEOREM}

In [2], Bombieri proved the following tauberian theorem by an elementary argument.

Theorem 3.1. Suppose that $a_{m} \geq 0$ and that

$$
m a_{m}+\sum_{i=1}^{m-1} a_{i} a_{m-i}=2 m+O(1)
$$

and

$$
\sum_{i=1}^{m} a_{2 i}=m+O(1)
$$

as $m \rightarrow \infty$. Then $a_{m} \sim 1$.

Remark. Instead of (3.2), Bombieri's hypothesis is

$$
m a_{2 m}+\sum_{i=1}^{m-1} a_{2 i} a_{2 m-2 i}=2 m+O(1)
$$

However, what he really needed is (3.2), which is a consequence of (3.3).

We can refine this theorem in the following quantitative form.

Theorem 3.2. Assume the hypothesis of Theorem 3.1. Then $a_{m}=1+O\left(\frac{1}{m}\right)$ as $m \rightarrow \infty$.

Remark. Let $a_{m}=1$ if $m$ is not a square of an integer and $a_{n^{2}}=1+1 / n^{2}$. Then, plainly (3.1) and (3.2) are satisfied. This example shows that $a_{m}=$ $1+O\left(\frac{1}{m}\right)$ as $m \rightarrow \infty$ is sharp.

Proof of Theorem 3.2. We first note that (3.1) implies

$$
\sum_{i=1}^{m} a_{i}=m+O(1)
$$

by a tauberian theorem of Erdös [5]. Following [2], we set $a_{m}=1+r_{m}$. It suffices to show $r_{m}=O\left(\frac{1}{m}\right)$. From (3.1) and (3.4), we have

$$
m r_{m}+\sum_{k=1}^{m-1} r_{k} r_{m-k}=O(1)
$$


and from Theorem 3.1, $r_{m} \rightarrow 0$ as $m \rightarrow \infty$. Therefore, there exists a constant $K$ such that

$$
\left|m r_{m}+\sum_{k=1}^{m-1} r_{k} r_{m-k}\right| \leq K
$$

holds for $m=1,2, \ldots$. We fix a positive integer $m_{0}$ sufficiently large so that, for $m \geq m_{0}$,

$$
\frac{1}{m} \sum_{k=1}^{m}\left|r_{k}\right|<\frac{1}{4}, \quad\left|r_{m}\right|<\frac{1}{4} .
$$

We shall show that for every positive integer $k$ if $m \geq 2^{k} m_{0}$ then

$$
\left|r_{m}\right| \leq\left(\frac{1}{4}\right)^{k+1}+\frac{K}{m}\left(1+\eta+\eta^{2}+\cdots+\eta^{k-1}\right)
$$

where

$$
\eta=\frac{1}{4}+\frac{3}{4} \log 2 \text {. }
$$

This leads to the conclusion of the theorem immediately. In fact, for any $m \geq$ $2 m_{0}$, if we take $k$ satisfying $2^{k+1} m_{0}>m \geq 2^{k} m_{0}$, from (3.8), we shall have

$$
\begin{aligned}
\left|r_{m}\right| & \leq 2^{-2 k-2}+\frac{K}{1-\eta} \frac{1}{m} \\
& \leq\left(\frac{m_{0}}{m}\right)^{2}+\frac{K}{1-\eta} \frac{1}{m} \leq\left(m_{0}+\frac{K}{1-\eta}\right) \frac{1}{m} .
\end{aligned}
$$

To prove (3.8), we first note that, for $k=1,(3.8)$ is certainly true. Actually, we have, from (3.6) and (3.7),

$$
\begin{aligned}
\left|r_{m}\right| & \leq \frac{1}{m}\left\{2 \sum_{s=1}^{[m / 2]}\left|r_{s}\right|\left|r_{m-s}\right|+K\right\} \\
& \leq \frac{1}{m}\left\{\frac{1}{2} \sum_{s=1}^{[m / 2]}\left|r_{s}\right|+K\right\} \leq\left(\frac{1}{4}\right)^{2}+\frac{K}{m} .
\end{aligned}
$$

Therefore, we assume (3.8) for $1 \leq k \leq l$ and consider $m \geq 2^{l+1} m_{0}$. We denote $\eta_{k}=1+\eta+\eta^{2}+\cdots+\eta^{k-1}$. Then we have, by the induction hypothesis,

$$
\begin{aligned}
\left|r_{m}\right| & \leq \frac{1}{m}\left\{2 \sum_{s=1}^{[m / 2]}\left|r_{s}\right|\left|r_{m-s}\right|+K\right\} \\
& \leq \frac{1}{m}\left\{2 \sum_{s=1}^{[m / 2]}\left|r_{s}\right|\left(\left(\frac{1}{4}\right)^{l+1}+\frac{K}{m-s} \eta_{l}\right)+K\right\} \\
& =\frac{2}{m}\left(\frac{1}{4}\right)^{l+1} \sum_{s=1}^{[m / 2]}\left|r_{s}\right|+\frac{2 K}{m} \eta_{l} \sum_{s=1}^{[m / 2]}\left|r_{s}\right| \frac{1}{m-s}+\frac{K}{m}
\end{aligned}
$$

since, for $s \leq\left[\frac{m}{2}\right], m-s \geq \frac{m}{2} \geq 2^{l} m_{0}$. We shall estimate the last two sums in (3.10) separately. 
First, we have, by (3.7),

$$
\begin{aligned}
\sum_{s=1}^{[m / 2]}\left|r_{s}\right| \frac{1}{m-s} & =\sum_{s=1}^{\left[2^{-l-1} m\right]}\left|r_{s}\right| \frac{1}{m-s}+\sum_{s=\left[2^{-l-1} m\right]+1}^{[m / 2]}\left|r_{s}\right| \frac{1}{m-s} \\
& \leq \frac{1}{4}\left(\frac{1}{m-2^{-l-1} m} 2^{-l-1} m+\sum_{s=\left[2^{-l-1} m\right]+1}^{[m / 2]} \frac{1}{m-s}\right) \\
& \leq \frac{1}{4}\left\{\frac{1}{3}+\log 2\right\}=\frac{1}{3} \eta
\end{aligned}
$$

since

$$
\sum_{s=\left[2^{-l-1} m\right]+1}^{[m / 2]} \frac{1}{m-s}=\sum_{k=m-[m / 2]}^{m-\left[2^{l-1} m\right]-1} \frac{1}{k} \leq \int_{m / 2-1}^{m-2} \frac{d t}{t}=\log 2 .
$$

Hence,

$$
\frac{2 K}{m} \eta_{l} \sum_{s=1}^{[m / 2]}\left|r_{s}\right| \frac{1}{m-s} \leq \frac{2}{3} \frac{K}{m} \eta_{l} \eta .
$$

Secondly, we have, by (3.7) and (3.8) with $1 \leq k \leq l$,

$$
\begin{aligned}
\sum_{s=1}^{[m / 2]}\left|r_{s}\right|= & \sum_{s=1}^{\left[2^{-l} m\right]}\left|r_{s}\right|+\sum_{s=\left[2^{-l} m\right]+1}^{\left[2^{-l+1} m\right]}\left|r_{s}\right|+\cdots+\sum_{s=[m / 4]+1}^{[m / 2]}\left|r_{s}\right| \\
\leq & {\left[2^{-l} m\right] \frac{1}{4}+\sum_{s=\left[2^{-l} m\right]+1}^{\left[2^{-l+1} m\right]}\left(\left(\frac{1}{4}\right)^{2}+\frac{K}{s} \eta_{1}\right) } \\
& +\sum_{s=\left[2^{-l+1} m\right]+1}^{\left[2^{-l+2} m\right]}\left(\left(\frac{1}{4}\right)^{3}+\frac{K}{s} \eta_{2}\right)+\cdots+\sum_{s=[m / 4]+1}^{[m / 2]}\left(\left(\frac{1}{4}\right)^{l}+\frac{K}{s} \eta_{l-1}\right) \\
\leq & \frac{1}{4}\left\{\left[2^{-l} m\right]+\left(\left[2^{-l+1} m\right]-\left[2^{-l} m\right]\right) \frac{1}{4}\right. \\
& \left.+\left(\left[2^{-l+2} m\right]-\left[2^{-l+1} m\right]\right)\left(\frac{1}{4}\right)^{2}+\cdots+\left(\left[\frac{m}{2}\right]-\left[\frac{m}{4}\right]\right)\left(\frac{1}{4}\right)^{l-1}\right\} \\
& +K \eta_{l-1} \sum_{s=\left[2^{-l} m\right]+1}^{[m / 2]} \frac{1}{s} \\
= & \frac{1}{4} \Sigma_{1}+K \eta_{l-1} \sum_{s=\left[2^{-l} m\right]+1}^{[m / 2]} \frac{1}{s},
\end{aligned}
$$

say. Therefore, if $l=1$, we have $\sum_{s=1}^{[m / 2]}\left|r_{s}\right| \leq m 2^{-l} / 4$. If $l \geq 2$, we have

$$
\begin{aligned}
\Sigma_{1}= & \frac{3}{4}\left[2^{-l} m\right]+\frac{3}{4} \frac{1}{4}\left[2^{-l+1} m\right]+\frac{3}{4}\left(\frac{1}{4}\right)^{2}\left[2^{-l+2} m\right] \\
& +\cdots+\frac{3}{4}\left(\frac{1}{4}\right)^{l-2}\left[\frac{m}{4}\right]+\left(\frac{1}{4}\right)^{l-1}\left[\frac{m}{2}\right] \\
\leq & \frac{3}{4} 2^{-l} m\left(1+\frac{1}{2}+\frac{1}{2^{2}}+\cdots+\frac{1}{2^{l-2}}\right)+\left(\frac{1}{4}\right)^{l-1} \frac{m}{2} \\
\leq & \frac{3}{2} 2^{-l} m+\left(\frac{1}{4}\right)^{l-1} \frac{m}{2}
\end{aligned}
$$


and

$$
\sum_{s=[2-l m]+1}^{[m / 2]} \frac{1}{s}<\int_{\left[2^{-l} m\right]}^{[m / 2]} \frac{d t}{t} \leq \log \frac{m / 2}{\left[m / 2^{l}\right]} \leq l \log 2
$$

since $m \geq 2^{l+1} m_{0}>2^{l+1}$. Therefore, from (3.12),

$$
\begin{aligned}
\sum_{s=1}^{[m / 2]}\left|r_{s}\right| & \leq \frac{1}{4}\left\{\frac{3}{2} 2^{-l} m+\left(\frac{1}{4}\right)^{l-1} \frac{m}{2}\right\}+K \eta_{l-1} l \log 2 \\
& \leq \frac{m}{4} 2^{-l+1}+K \eta_{l-1} l \log 2
\end{aligned}
$$

Hence, if $l \geq 2$,

$$
\begin{aligned}
\frac{2}{m}\left(\frac{1}{4}\right)^{l+1} \sum_{s=1}^{[m / 2]}\left|r_{s}\right| & \leq\left(\frac{1}{4}\right)^{l+2}+\frac{K}{m} \eta_{l-1} \frac{2 l \log 2}{4^{l+1}} \\
& \leq\left(\frac{1}{4}\right)^{l+2}+\frac{1}{16} \frac{K}{m} \eta_{l-1} \eta .
\end{aligned}
$$

Plainly, (3.13) is true if $l=1$.

Now, applying (3.11) and (3.13) to (3.10), we arrive at

$$
\begin{aligned}
\left|r_{m}\right| & \leq\left(\frac{1}{4}\right)^{l+2}+\frac{1}{16} \frac{K}{m} \eta_{l-1} \eta+\frac{2}{3} \frac{K}{m} \eta_{l} \eta+\frac{K}{m} \\
& \leq\left(\frac{1}{4}\right)^{l+2}+\frac{K}{m} \eta_{l+1} .
\end{aligned}
$$

This completes the proof of (3.8) and hence the proof of the theorem.

\section{AN ELEMENTARY PROOF OF THE ABSTRACT PRIME NUMBER THEOREM}

We can now give elementary proofs of the following two theorems.

Theorem 4.1. Suppose there exist constants $A>0, B>0, q>1, \gamma>3$, and $\delta>1$ such that

$$
G(n)=A q^{n}+O\left(q^{n} n^{-\gamma}\right), \quad n=1,2, \ldots,
$$

and

$$
\sum_{k=0}^{2 n}(-1)^{k} G(k) G(2 n-k)=B q^{2 n}+O\left(q^{2 n} n^{-\delta}\right), \quad n=1,2, \ldots
$$

Then

$$
\Lambda(n)=q^{n}+O\left(\frac{q^{n}}{n}\right)
$$

Theorem 4.2. Suppose there exist constants $A>0$ and $q>1$ such that $\sum_{n=1}^{\infty}\left(G(n)-A q^{n}\right)^{2} q^{-n}<\infty$. Then (4.1) holds. 
Remark 1. The asymptotic result $\Lambda(n) \sim q^{n}$ (or in his notation, $N_{m} \sim q^{m}$, the purely asymptotic conclusion of a well-known theorem of A. Weil) was first stated by Bombieri [1] for an algebraic curve $C$. An analytic proof was given by the author in [8]. In a recent paper [2], Bombieri proved $N_{m} \sim q^{m}$ for $C$ by means of the elegant tauberian theorem quoted in Theorem 3.1 and formulas (1.5) and (2.3). He proved (1.5) and (2.3) by using some special properties of the curve $C$. Knopfmacher's elementary proof [6] of $\Lambda(m) \sim q^{m}$, following the proof in [1], was defective.

Remark 2. Theorem 4.1 is a new version of the abstract prime number theorem for algebraic function fields. Theorem 4.2 is a weaker form of Theorem 3.6 in [8]. There are simple examples to which Theorem 4.1 applies but Theorem 4.2 does not.

Proof of Theorems 4.1 and 4.2. The hypothesis of Theorem 4.1 implies, by Theorem 1.2 and Lemma 2.2,

$$
m \frac{\Lambda(m)}{q^{m}}+\sum_{k=1}^{m-1} \frac{\Lambda(k)}{q^{k}} \frac{\Lambda(m-k)}{q^{m-k}}=2 m+O(1)
$$

and

$$
\sum_{k=1}^{m} \frac{\Lambda(2 k)}{q^{2 k}}=m+O(1)
$$

Also, the hypothesis of Theorem 4.2 implies, by Theorem 1.2 and Theorem 2.1 , the above formulas. The same conclusions of Theorems 4.1 and 4.2 follow immediately by taking $a_{m}=\Lambda(m) q^{-m}$ in Theorem 3.2.

Combining Theorem 4.1 and Theorems 2.1 and 2.5 in [8] with analytic argument, the next theorem follows directly.

Theorem 4.3. If the first condition of Theorem 4.1 is replaced by $G(n)=A q^{n}+$ $O\left(q^{\nu_{n}}\right), n=1,2, \ldots$ with $0<\nu<1$, then

$$
\Lambda(n)=q^{n}+O\left(q^{\theta_{n}}\right)
$$

holds for some $\theta$ with $\nu<\theta<1$.

\section{ANOTHER ELEMENTARY PROOF OF THE ABSTRACT PRIME NUMBER THEOREM}

To conclude our discussion, we shall show briefly that the original idea in [1] leads to another proof of Theorem 3.1. However, this proof seems now clumsy by comparison with Bombieri's elegant proof [2]. In particular, this gives another elementary proof of the P.N.T.

As in [1], we set $a_{m}=1+r_{m}$. From (3.1), (3.2), and (3.4), we have

$$
\begin{gathered}
m r_{m}+\sum_{k=1}^{m-1} r_{k} r_{m-k}=O(1), \\
\left|r_{m}\right| \leq 1+O\left(\frac{1}{m}\right) \\
\sum_{k=1}^{m} r_{k}=O(1)
\end{gathered}
$$


and

$$
\sum_{k=1}^{m} r_{2 k}=O(1)
$$

Then, from (5.2) and (5.1), lim $\sup _{m \rightarrow \infty}\left|r_{m}\right|=A \leq 1$ and $A \leq A^{2}$, hence either $A=0$ or $A=1$. It suffices to show $A<1$.

To this end, first, we can prove the following lemma which is a strengthened form of the lemma in Bombieri's paper [2]. The proof follows his general idea.

Lemma 5.1. We have

$$
\limsup _{m \rightarrow \infty}\left|r_{m}-r_{m+1}\right| \leq 1, \quad \limsup _{m \rightarrow \infty}\left|r_{m}+r_{m+1}\right| \leq 1
$$

(however, only the first inequality is needed in what follows).

Remark. Lemma 5.1 shows that $\left\{r_{m}\right\}$ has a kind of slow oscillation which is essential for deducing Theorem 3.1 from the following lemma of Wirsing (see $[3,7])$.

Lemma 5.2. Let $f$ and $g$ be real-valued Lebesgue measurable functions defined on $[0, \infty)$, which are square-integrable over every finite subinterval. Let

$$
\limsup _{x \rightarrow \infty} \frac{1}{x} \int_{0}^{x} f^{2}(y) d y=F, \quad \limsup _{x \rightarrow \infty} \frac{1}{x} \int_{0}^{x} g^{2}(y) d y=G,
$$

where $F$ and $G$ are finite. Let $h$ be the function defined by

$$
h(x)=\frac{1}{x} \int_{0}^{x} f(x-y) g(y) d y, \quad x>0 .
$$

If $\lim _{x \rightarrow \infty} \frac{1}{x} \int_{0}^{x} h(y) d y=0$, then

$$
\limsup _{x \rightarrow \infty} \frac{1}{x} \int_{x_{0}}^{x} h^{2}(y) d y \leq \frac{1}{2} F G
$$

where $x_{0}$ is any positive number.

Sketch of a proof of Theorem 3.1 by using Wirsing's lemma. We apply Lemma 5.2 to the functions $f(x)=g(x)=r_{k}$, for $k-1 \leq x<k$. It is easy to show that

$$
\begin{aligned}
h(x) & =\frac{1}{x} \sum_{k=1}^{[x]} r_{k} \int_{k-1}^{k} f(x-y) d y+O\left(\frac{1}{x}\right) \\
& =\frac{\{x\}}{x}([x]+2) r_{[x]+2}+\frac{1-\{x\}}{x}([x]+1) r_{[x]+1}+O\left(\frac{1}{x}\right)
\end{aligned}
$$

by (5.1) and (5.2). Therefore, we have

$$
\frac{1}{x} \int_{0}^{x} h(y) d y=\frac{1}{x}\left\{O(\log x)+\sum_{k=1}^{[x]} r_{k}\right\} \rightarrow 0
$$

as $x \rightarrow \infty$. Thus, the convolution average hypothesis of Wirsing's lemma is satisfied. Moreover, by using (5.6), a careful calculation yields

$$
\frac{1}{x} \int_{1}^{x} h^{2}(y) d y=\frac{1}{x}\left\{\frac{2}{3} \sum_{k=1}^{[x]} r_{k}^{2}+\frac{1}{3} \sum_{k=1}^{[x]} r_{k} r_{k+1}+O(\log x)\right\} \text {. }
$$


Now, by the Cauchy-Schwarz inequality, we have

$$
\begin{aligned}
\sum_{k=1}^{[x]} r_{k} r_{k+1} & =\sum_{k=1}^{[x]} r_{k}^{2}-\sum_{k=1}^{[x]} r_{k}\left(r_{k}-r_{k+1}\right) \\
& \geq \sum_{k=1}^{[x]} r_{k}^{2}-\left(\sum_{k=1}^{[x]} r_{k}^{2}\right)^{1 / 2}\left(\sum_{k=1}^{[x]}\left(r_{k}-r_{k+1}\right)^{2}\right)^{1 / 2},
\end{aligned}
$$

and hence

$$
\begin{aligned}
\frac{1}{x} \int_{1}^{x} h^{2}(y) d y \geq & \frac{1}{x} \sum_{k=1}^{[x]} r_{k}^{2}-\frac{1}{3}\left(\frac{1}{x} \sum_{k=1}^{[x]} r_{k}^{2}\right)^{1 / 2} \\
& \times\left(\frac{1}{x} \sum_{k=1}^{[x]}\left(r_{k}-r_{k+1}\right)^{2}\right)^{1 / 2}+O(\log x) .
\end{aligned}
$$

Set

$$
\limsup _{m \rightarrow \infty} \frac{1}{m} \sum_{k=1}^{m} r_{k}^{2}=B
$$

Note that $B \leq 1$ by (5.2). Then, by Wirsing's lemma, we arrive at $B-\frac{1}{3} \sqrt{B} \leq$ $B^{2} / 2$, since, by Lemma 5.1 ,

$$
\limsup _{m \rightarrow \infty}\left(\frac{1}{m} \sum_{k=1}^{m}\left(r_{k}-r_{k+1}\right)^{2}\right)^{1 / 2} \leq 1 .
$$

It follows that $B<1$.

Finally, by (5.1) and the Cauchy-Schwarz inequality, we have

$$
\begin{aligned}
A & =\limsup _{m \rightarrow \infty}\left|r_{m}\right|=\limsup _{m \rightarrow \infty} \frac{1}{m}\left|\sum_{k=1}^{m-1} r_{k} r_{m-k}\right| \\
& \leq \limsup _{m \rightarrow \infty} \frac{1}{m} \sum_{k=1}^{m} r_{k}^{2}=B<1 .
\end{aligned}
$$

This completes the proof.

\section{REFERENCES}

1. E. Bombieri, Sull'analogo della formula di Selberg nei corpi di funzioni, Atti Accad. Naz. Lincei Rend. Cl. Sci. Fis. Mat. Natur. (8) 35 (1963), 252-257.

2. __ Correction to my paper "Sull'analogo della formula di Selberg nei corpi di funzioni" (preprint).

3. K. Chandrasekharan, Arithmetic functions, Springer-Verlag, 1970.

4. H. G. Diamond, Elementary methods in the study of the distribution of prime numbers, Bull. Amer. Math. Soc. (N.S.) 1 (1982), 553-589.

5. P. Erdös, On a tauberian theorem connected with the new proof of the prime number theorem, J. Indian Math. Soc. 13 (1944), 133-147. 
6. J. Knopfmacher, An abstract prime number theorem relating to algebraic function fields, Arch. Math. 29 (1977), 271-279.

7. E. Wirsing, Elementare Beweise des Primzahlsatzes mit Restglid. II. J. Reine Angew. Math. 214/215 (1964), 1-18.

8. W.-B. Zhang, The abstract prime number theorem for algebraic function fields, Analytic Number Theory, Proc. Conf. in Honor of Paul T. Bateman (Allerton Park, 1989), BirkhäuserVerlag, 1990, pp. 529-558.

Department of Mathematics, University of Illinois, 1409 West Green Street, Urbana, ILLINOIS 61801

E-mail address:wbzhang@symcom.math.uiuv.edu 